\title{
Embedded Object-Oriented Micromagnetic Frame (OOMMF) for More Flexible Micromagnetic Simulations
}

\author{
Hyungsuk Kim ${ }^{1}$ and Chun-Yeol You ${ }^{2 *}$ \\ ${ }^{1}$ Department of Electrical Engineering, Kwangwoon University, Seoul 01897, Korea \\ ${ }^{2}$ Department of Emerging Materials Science, Daegu Gyeongbuk Institute of Science and Technology, Daegu 42988, Korea
}

(Received 15 November 2016, Received in final form 6 December 2016, Accepted 6 December 2016)

\begin{abstract}
We developed an embedded Object-Oriented Micromagnetic Frame (OOMMF) script schemes for more flexible simulations for complex and dynamic mircomagnetic behaviors. The OOMMF can be called from any kind of softwares by system calls, and we can interact with OOMMF by updating the input files for next step from the output files of the previous step of OOMMF. In our scheme, we set initial inputs for OOMMF simulation first, and run OOMMF for $\Delta t$ by system calls from any kind of control programs. After executing the OOMMF during $\Delta t$, we can obtain magnetization configuration file, and we adjust input parameters, and call OOMMF again for another $\Delta t$ running. We showed one example by using scripting embedded OOMMF scheme, tunneling magneto-resistance dependent switching time. We showed the simulation of tunneling magneto-resistance dependent switching process with non-uniform current density using the proposed framework as an example.
\end{abstract}

Keywords : micromagnetic simulation, spin transfer torque, magnetic random access memory (MRAM), tunneling magneto-resistance

\section{Introductions}

Micromagnetic simulation is one of the essential and powerful tools in magnetism researches. Since the idea of micromagnetics has been developed by Brown and Aharoni [1, 2], there are tremendous improvements in the micromagnetic simulations. By accompanying unbelievable increment of computation power, and shrink down of size of nano spin devices, the micromagnetic simulation shows excellent agreement with experiments, and it became more important in order to get better understanding and insight of the complex spin dynamics. In the history of micromagnetics, one of the remarkable events is launching of Object-Oriented Micromagnetic Frame (OOMMF) [3], which is open source based. Before OOMMF, researchers must develop their own code, and it was a re-invention of the wheel. After OOMMF, it is easy for most researchers to confirm their experiments or test their ideas by micromagnetic simulations. After that there are more free or commercial micromagnetic simulators

CThe Korean Magnetics Society. All rights reserved.

*Corresponding author: Tel: +82-53-785-6522

Fax: +82-53-785-6509, e-mail: cyyou@dgist.ac.kr are available [4-8]. Since OOMMF and other public micromagnetic simulators such as $\operatorname{MuMax}^{3}[4]$ are open source, it is possible to extend and modify the code, so there are many extension modules are available in public domain [9]. However, modifying source code requires a lot of effort and deep understanding of both physics of micromagnetics and programming. Even though, the existing micromagnetic simulators are very flexible, and they can handle many practical cases, sometimes we need to simulate more realistic complex problems, where the materials' parameters are changed because of the temperature, or the resistances are changed because of the spin configurations and magneto-resistance. Such simulation requires more flexible control of the simulator.

In this work, we propose an effective framework to perform micromagnetic simulations for more flexible way. In our scheme, we used OOMMF as micromagnetic simulator and MatLab as main control program language, however, the proposed idea is applicable with the combination of any micromagnetic simulator which supports script mode running such as OOMMF [3] and $\mathrm{MuMax}^{3}$ [4], and any kind of programming languages who can call system command. The main idea of this work is shown in Fig. 1 as pseudo code flow chart. First, we set up initial 


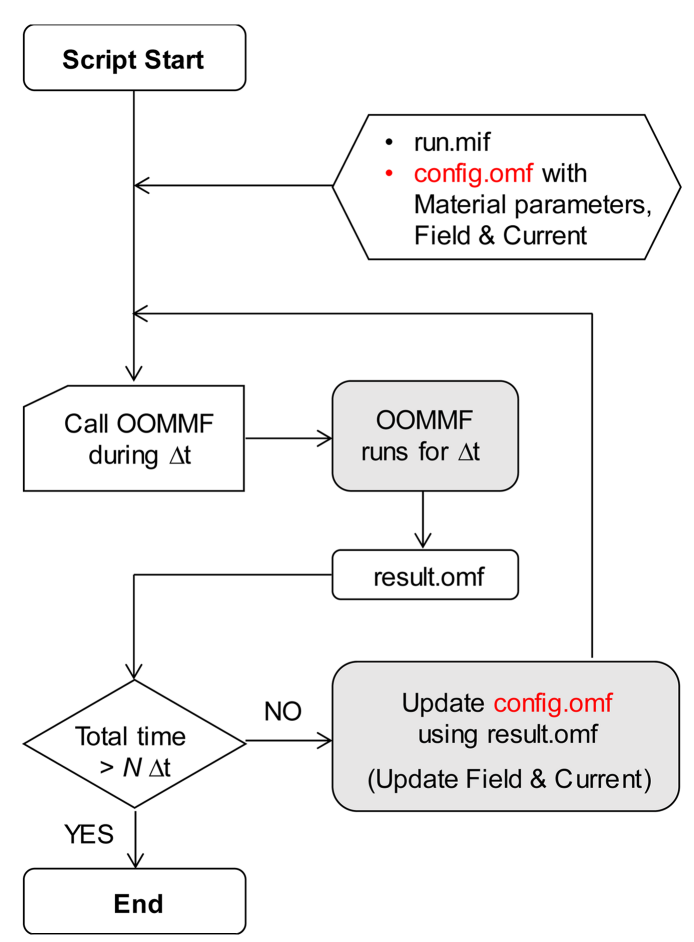

Fig. 1. (Color online) Schematic flow chart of the simulations. We start main code with previously defined *.mif and *.omf, which is required for OOMMF running. After running OOMMF during $\Delta t$, OOMMF generates updated spin configuration file. Main program reads the spin configuration file, and determines new parameters which depend on previous results. With updated initial file, the main program calls OOMMF again, and repeats those procedures. The main program will terminate the simulation when the spin configuration satisfies specific conditions, or the total simulation time is reached.

configuration files (*.omf file) for the magnetization (or spin) for a given problem and problem definition file (*.mif for OOMMF, *.mx3 for $\mathrm{MuMax}^{3}$ ). Using the control programming language, we can edit the problem definition file or configuration files for the simulators any time. At the first step, we call the system command and run the micromagnetic executable for a short time $\Delta t$ with given materials' parameters, external magnetic field, and current (for spin transfer torque simulations). After running the simulation for $\Delta t$, we obtained new micromagnetic configuration file (*.omf) as a result of the simulation. The obtained file can be used to update the initial configuration file in the control program. We also calculate some materials' parameters and/or current density which depends on the spin configuration of the given problem. And we may edit problem definition file also, if we need. After this procedure, we call micromagnetic executable again for another $\Delta t$. Here it is not necessary to use the same $\Delta t$, it also can be adjusted if we need. We can repeat the procedure till when the total simulation time is satisfied. With this idea, we can make micromagnetic simulation more flexible, without modifying micromagnetic simulator itself.

\section{Current Induced Magnetization Switching for PMA with Non-Uniform Current Density Distribution}

In this work, we will show an example. We simulate the current induced magnetization switching for perpendicular magnetic anisotropy (PMA) system which is important in the STT-MRAM (spin transfer torque magnetoresistive random access memory). Series of micromagnetic simulations for STT had been already published by using "CYY_STTEvolve" extension module [10-13]. However, all of those simulations assumed uniform current density (UCD) during the switching process. In general, the tunneling magneto-resistance (TMR) is more than 100 $\%$, it implies the resistance between parallel $(\mathrm{P})$ state and anti-parallel (AP) state is quite different. Furthermore, in practical circuits for STT-MRAM devices, the magnetic tunneling junction (MTJ) is usually operated by a constant voltage mode. Therefore, actual current density of MTJ will depend on the spin configurations. When the spin configuration is P-state, the resistance is smaller (larger current), while AP-state has larger resistance (smaller current) for a constant voltage operation mode. Furthermore, during the switching process, the spin configurations varies from point to point, so that the local current density for each point will be different. With our best knowledge, only one group reported the effect of the nonuniform current density for STT switching for in-plane magnetization case [14]. They found that non-uniform current density (NUCD) played important role in the details of spin dynamics, using their own micromagnetic code. Since they studied the effect of the NUCD by their own code, it is not available to test it for other groups. Here, we present how we can implement the effect of NUCD for PMA MTJ, and we performed it by using public domain micromagnetic simulator OOMMF, it can be tested by anyone.

\section{Details of the Simulations}

Figure 2 shows the schematic sample structure which we examined in this study. The MTJ consists of three layer, polarizer (or fixed, or reference) layer $(1 \mathrm{~nm})$, insulator layer $(1 \mathrm{~nm})$, and free (or switching) layer (1 $\mathrm{nm}$ ). We assumed fixed and free layer have uniaxial PMA energies, 2.6 and $1.3 \times 10^{6} \mathrm{~J} / \mathrm{m}^{3}$, respectively. The satura- 
(a)

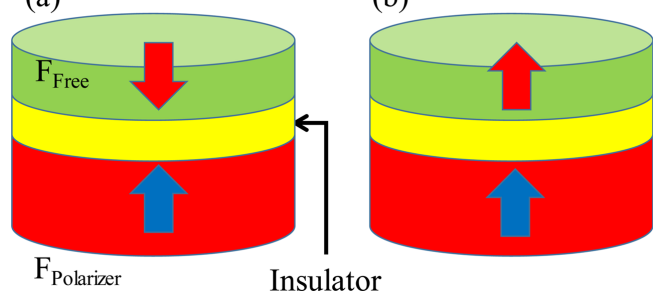

Fig. 2. (Color online) Typical sample structures of MTJ. (a) anti-parallel configuration and (b) parallel configuration.

tion magnetization of both layer is $1.3 \times 10^{6} \mathrm{~A} / \mathrm{m}$, the exchange stiffness constant is $30 \mathrm{pJ} / \mathrm{m}$, and damping constant is 0.02 , respectively. We set polarizer layer PMA energy is large enough in order to fix the spin orientation during the switching. The diameter of MTJ is $40 \mathrm{~nm}$, and each unit cell is $1 \times 1 \times 1 \mathrm{~nm}^{3}$. Here, we considered only Slonczewski STT [15] for the simplicity, and ignored field-like torque term [11]. We set $\eta_{p}$ is 0.7 for our simulations, where $\eta_{p}$ is spin polarization of the polarizer layer, or spin torque efficiency. In our previous studies and most of micromagnetic simulations, they don't need TMR values, since the switching behavior is not directly related with TMR values. However, in order to handle NUCD effect, the finite TMR value must be setup in the simulations. If the spin configuration is non-uniform, the local resistance will be varied by the large TMR, and it causes non-uniform current density for a constant voltage operation. If the local spin is anti-parallel (parallel), the local resistance will be large (small), and the current will be smaller (larger), so that the STT is smaller (larger). Therefore, the details of spin dynamics cannot be the same with the UCD model. In order to address the effect of NUCD, we have to update the local current density profile for each step, it will be a good example to test our embedded micromagnetic simulation scheme.

First, we set the initial condition of P- and AP-state with zero current, and run the micromagnetic simulation for a long enough time to find total energy minimization. With relaxed initial spin configuration, we initially applied uniform current density for short time $\Delta t(=10 \sim 100 \mathrm{ps})$. The OOMMF is called as a subroutine in our MatLab code by "boxsi" with proper options and parameters [3]. After running OOMMF for $\Delta t_{1}$, OOMMF generates spin configuration file. We used this output spin configuration file as initial spin configuration for the next step. Before calling the OOMMF again, we have to calculate the local non-uniform current density profile (see the next session). We calculate the local resistance and current density from the spin configuration file in the MatLab code. Now, we are ready to run OOMMF for next time step $\Delta t_{2}$. Gene- rally, there is no reason to set $\Delta t_{2}=\Delta t_{1}$. We can repeat this procedure till $t_{\text {total }}=\sum_{k} \Delta t_{k}$ or we may stop the simulation after checking the spin configurations for each step.

\section{Setup Non-Uniform Current Density Profile}

The procedure of the local current density profile is as follows. In the micromagnetic simulations, the real sample is divided by finite size unit cells, and we assumed that each $(i, j)$ cell of free layer is connected to corresponding bottom layer cell by insulator layer cell, and the tunneling current is mainly passed through the vertical path as shown in Fig. 3. Therefore, the resistance of each $(i, j)$ cell $R_{i, j}$ can be modeled by

$$
R_{i, j}=\frac{R_{\perp}}{1+\frac{T M R}{2} \cos \theta_{i, j}} .
$$

Here, $\theta_{i, j}$ is the angle between spins of free and polarizer layers of $(i, j)$ cell, and it can be calculated from the spin configurations of each layer in the micromagnetic simulations. $R_{\perp}$ is the resistance when $\theta=\pi / 2$. Therefore, our strategy is update the resistance of each cell for a given spin configuration. From the local resistance, we can calculate local current density:

$$
J_{i, j}=\frac{V}{R_{i, j}(\Delta x \Delta y)} .
$$

In this study, we used the definition of TMR as follows [16]:

$$
T M R \equiv \frac{2\left(G_{P}-G_{A P}\right)}{G_{P}+G_{A P}}
$$

Fig. 3. (Color online) Schematic diagram of parallel resistance model for MTJ. Each cell of MTJ is connect by parallel, and the tunneling between polarizer and free layer are modeled a resistance, whose value is a function of the angle between spins. 
Here, $G_{P}$ and $G_{A P}$ is conductance of P- and AP-state, respectively. The tunnel conductance is given by

$$
\begin{aligned}
& G=G_{P} \cos ^{2} \frac{\theta}{2}+G_{A P} \sin ^{2} \frac{\theta}{2}, \\
& R=\frac{1}{G}=\frac{R_{\perp}}{1+\frac{T M R}{2} \cos \theta} .
\end{aligned}
$$

It differs from well-known formula,

$$
R=R_{\perp}\left(1-\frac{T M R}{2} \cos \theta\right)
$$

Even though Eq. (6) is widely used in community, it has been revealed that it is correct only when TMR is small [16]. Since we focused on the high TMR case $(>100 \%)$ for realistic case for STT-MRAM, we take Eq.
(5) as a local resistance in our study.

In this study, we used "CYY_STTEvolve" extension module, however, the public version of this module can accept only uniform current density for whole sample area. In order to treat the effect of NUCD, we update the "CYY_STTEvolve" extension module to handle local current density profile [17]. In the updated extension module, the current density profile will be set by input file form or script form in the *.omf file.

\section{Results and Discussions}

We performed micromagnetic simulations for UCD and NUCD modes. Figure 4 (a) and (b) show the normalized magnetization of free layer as a function of the time for P- to AP-state and AP- to P-state switching for UCD and NUCD cases, respectively. We set the current density to
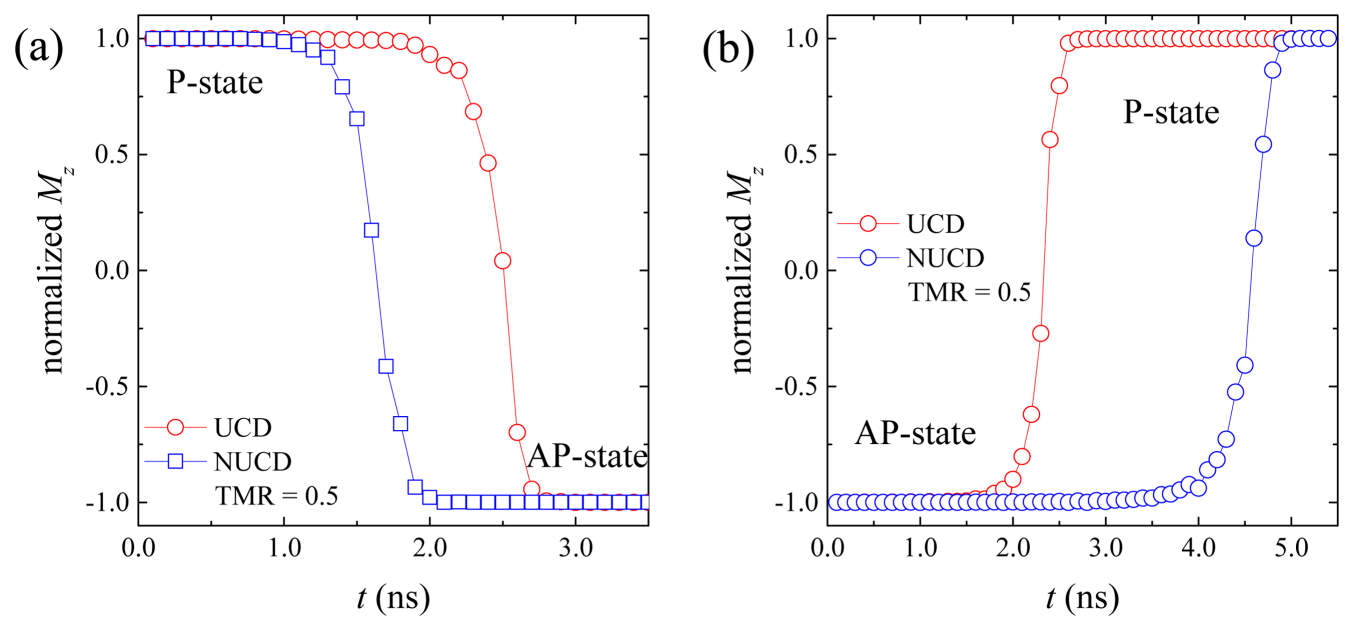

Fig. 4. (Color online) Normalized z-component magnetization of the free layer UCD and NUCD (TMR $=0.5$ ) modes for (a) P- to AP-state and (b) AP- to P-state switching processes.
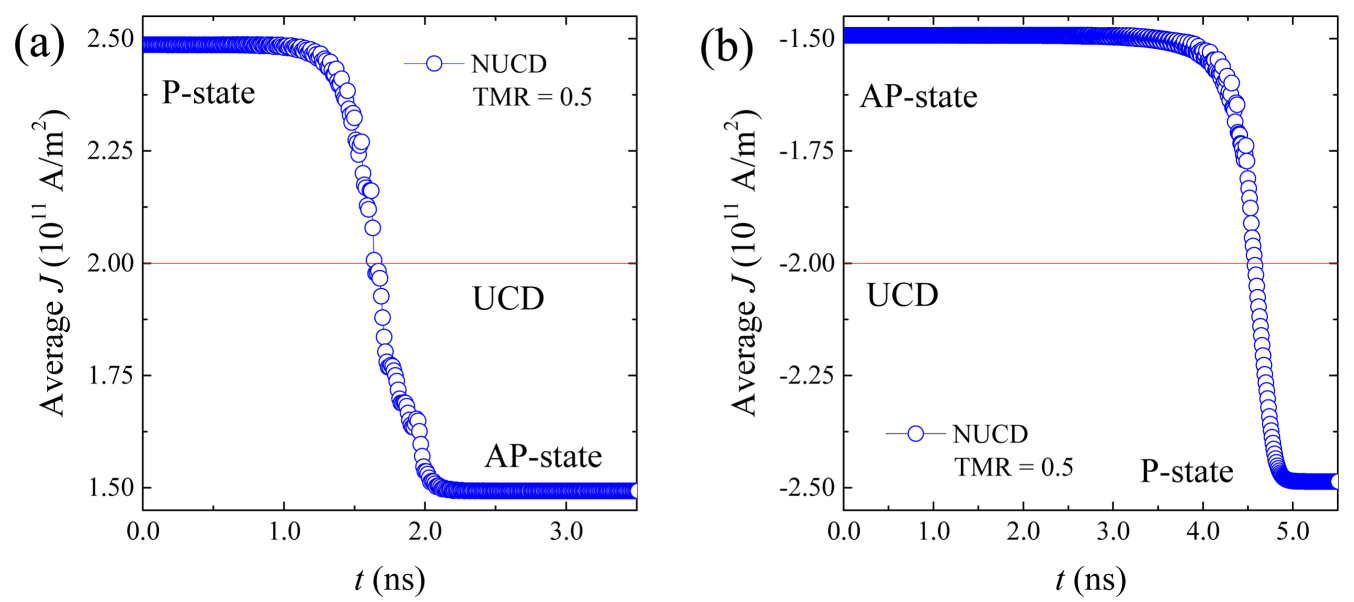

Fig. 5. (Color online) Average current density as a function of the time for (a) P- to AP-state and (b) AP- to P-state switching processes. The horizontal red solid line represents $\operatorname{UCD}\left(J=+/-2.0 \times 10^{11} \mathrm{~A} / \mathrm{m}^{2}\right)$. 
$+/-2.0 \times 10^{11} \mathrm{~A} / \mathrm{m}^{2}$ for UCD mode. For NUCD mode, we used the same current density when $\theta=\pi / 2$. It must be noted that the current density is varied as a function of the spin configurations and TMR values. We used TMR $=0.5$, which is corresponding to $100 \%$ of TMR for conventional definition $\left(R_{A P}-R_{P} / R_{P}\right)$. We find that the NUCD switching is faster (slower) than UCD mode for P- to AP-state (AP- to P-state) switching. In order to reveal the physical reason of different switching speed, we take a look more details of switching process. We plot the average current density of NUCD switching as a function of the time for $\mathrm{P}$ - to AP-state and AP- to P-state switching process in Fig. 5 (a) and (b). It is not surprising the absolute value of the average current density is larger (smaller) for P-state (AP-state) at the beginning, because the resistance of Pstate is smaller than AP-state by the finite TMR. Since we assumed constant voltage operation, the smaller resistance implies larger current density. Therefore, the P-state has larger current density for NUCD mode than UCD, it leads faster switching, while the AP-state has smaller current density. It is clearly shown in Fig. 5 (a) and (b). It must be addressed for the further study whether the only larger (smaller) current density is the origin of the faster (slower) switching or not. Since the NUCD will create more localized spin wave excitation, it may affect to the details of the switching process. More details spin dynamics will be studied elsewhere.

\section{Conclusions}

We implemented a MatLab code, where we can call "OOMMF" for short time running. The main code can communicate with OOMMF through input and output files by successive running of the "OOMMF" as an embedded command. With our proposed strategy, we can perform more flexible micromagnetic simulations. As an example, we presented STT switching with the finite TMR values, where the local current density is determined by the spin configurations. We believe that we pave the way of more flexible micromagnetic simulations. Our proposed method is applicable not only OOMMF, but also any kind of command line running simulators.

\section{Acknowledgements}

This work was supported by the grants from Kwangwoon University and from National Research Foundation of Korea (NRF) funded by the Korea government (Ministry of Education and Ministry of Science, ICT \& Future Planning) with the Grant Nos. of (Nos. NRF2015M3D1A107046, NRF-2015M2A2A6021171).

\section{References}

[1] W. F. Brown, "Micromagnetics". New York: Wiley (1963).

[2] A. Aharoni, "Introduction to the theory of Ferromagnetism", 2nd Ed. Oxford University Press (2000).

[3] M. J. Donahue and D. G. Porter, OOMMF User's Guide, Version 1.0, Interagency Report NISTIR 6376, National Institute of Standards and Technology, Gaithersburg, MD (Sept 1999).

[4] A. Vansteenkiste, J. Leliaert, M. Dvornik, M. Helsen, F. Garcia-Sanchez, and B. V. Waeyenberge, AIP Advances 4, 107133 (2014).

[5] M. Scheinfein, LLG Micromagnetic Simulator, http://llgmicro.home.mindspring.com/

[6] W. Scholz, Magpar, http://www.magpar.net/

[7] Vampire, http://vampire.york.ac.uk/features/

[8] Suessco Simulations, http://suessco.com/simulations/

[9] Extension modules for OOMMF, http://math.nist.gov/ oommf/contrib/oxsext

[10] Chun-Yeol You, Appl. Phys. Lett. 100, 252413 (2012).

[11] Chun-Yeol You, J. Magn. 17, 73 (2012).

[12] Chun-Yeol You, Appl. Phys. Expr. 5, 103001 (2012).

[13] Chun-Yeol You and Myung-Hwa Jung, J. Appl. Phys. 113, 073904 (2013).

[14] D. Aurélio, L. Torres, and G. Finocchio, J. Magn. Magn. Mater. 321, 3913 (2009).

[15] J. C. Slonczewski, J. Magn. Magn. Mater. L1, 159 (1996).

[16] H. Jaffrès, D. Lacour, F. Nguyen Van Dau, J. Briatico, F. Petroff, and a. Vaurès, Phys. Rev. B 64, 064427 (2001).

[17] The modified version of "CYY_STTEvolve" will be available upon request to author. 